\title{
High Thermoelectric Performance in Two-Dimensional Janus Monolayer Material WS-X $(X=$ Se and Te)
}

\author{
Abhishek Patel, Deobrat Singh,* Yogesh Sonvane, P. B. Thakor,* and Rajeev Ahuja* \\ Cite This: ACS Appl. Mater. Interfaces 2020, 12, 46212-46219 \\ Read Online
}

ABSTRACT: In the present work, Janus monolayers WSSe and WSTe are investigated by combining first-principles calculations and semiclassical Boltzmann transport theory. Janus WSSe and WSTe monolayers show a direct band gap of 1.72 and $1.84 \mathrm{eV}$ at $\mathrm{K}$-points, respectively. These layered materials have an extraordinary Seebeck coefficient and electrical conductivity. This combination of high Seebeck coefficient and high electrical conductivity leads to a significantly large power factor. In addition, the lattice thermal conductivity in the Janus monolayer is found to be relatively very low as compared to the $\mathrm{WS}_{2}$ monolayer. This leads to a high figure of merit (ZT) value of 2.56 at higher temperatures for the Janus WSTe monolayer. We propose that the Janus WSTe monolayer could be used as a potential thermoelectric
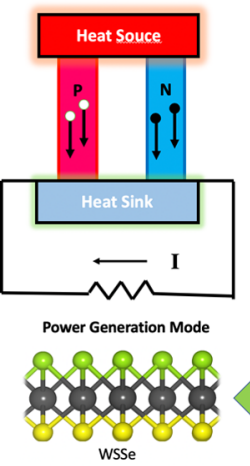

wsse

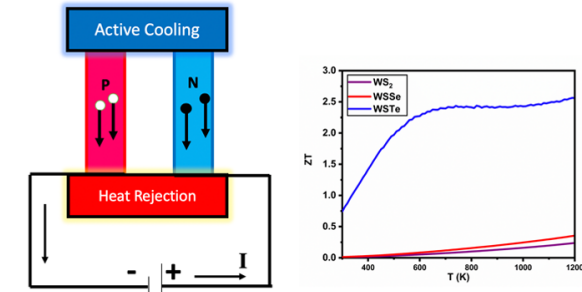

Refrigeration Mode

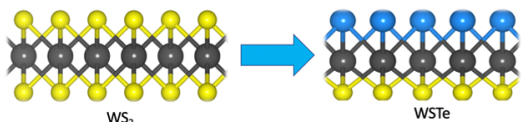
material due to its high thermoelectric performance. The result suggests that the Janus monolayer is a better candidate for excellent thermoelectric conversion.

KEYWORDS: 2D Janus monolayer, electronic properties, thermoelectric properties, lattice-thermal conductivity, Boltzmann transport equation

\section{INTRODUCTION}

The world's energy demand is rapidly increasing due to factors like considerable growth in population, industrialization, development in technology, etc. Until recently, these energy needs were being fulfilled with fossil fuels and other nonrenewable resources; ${ }^{1}$ however, the massive consumption of such resources led to the unavailability of them. The burning process generates energy with waste heat, exhaust gases, pollution, and other harmful effects on the environment. ${ }^{2,3}$ The renewable resources have a small contribution in fulfilling these energy needs. The total replacement of fossil fuels is not possible at present. ${ }^{4}$ Hence, improved energy efficiency and reduced energy demand are the expected solutions to this problem. ${ }^{5}$ Thermoelectric materials have the ability to convert electricity from heat by the Seebeck effect, and they are also able to produce a cooling effect by the Peltier effect. ${ }^{6}$ Lightweight, cost-effective, and efficient thermoelectric materials can help in generating electric energy from waste heat. Development and identification of such materials with high thermoelectric conversion performance have remained a big challenge since the discovery of the thermoelectric concept. ${ }^{7,8}$ The ability of thermoelectric conversion of thermoelectric material is summarized by the thermoelectric figure of merit $(\mathrm{ZT})$, which is expressed as $\mathrm{ZT}=S^{2} \sigma \mathrm{T} / \kappa$, where $S, \sigma$, and $\kappa$ represent the Seebeck coefficient, electrical conductivity, and thermal conductivity, respectively. ${ }^{9-11}$ The thermal conductivity of the material is expressed as $\kappa=\kappa_{\mathrm{l}}+\kappa_{\mathrm{e}}$. The thermal conductivity $(k)$ involves lattice thermal conductivity $\left(\kappa_{1}\right)$ due to lattice vibrations and electronic contributions $\left(\kappa_{\mathrm{e}}\right)$ due to electronic motion. ${ }^{10,11}$ The best thermoelectric performance measured until now has $\mathrm{ZT}$ values ranging from 2.5 to 2.8. ${ }^{11-14}$ The new material (a thin layer of iron, vanadium, tungsten, and aluminum applied to a silicon crystal) developed by the scientists of TU Wien has shown that the high efficiency $\mathrm{ZT}$ value lies between 5 and $6 .^{15}$

The new derivatives of two-dimensional (2D) materials, Janus 2D materials, have attracted considerable research attention due to their distinct properties. Their properties are quite different compared to the traditional $2 \mathrm{D}$ materials. ${ }^{16}$ The Janus 2D material possesses unique properties due to its mirror asymmetry in the structure. ${ }^{16,17}$ The Janus transition metal dichalcogenides (TMDs) belong to an important class of materials due to their structure, stability, electronic properties, and other properties. ${ }^{16}$ In 2013, the dynamical stability of MoSSe, WSSe, WSTe, and WSeTe was proved through a

Received: August 3, 2020

Accepted: September 15, 2020

Published: September 15, 2020 

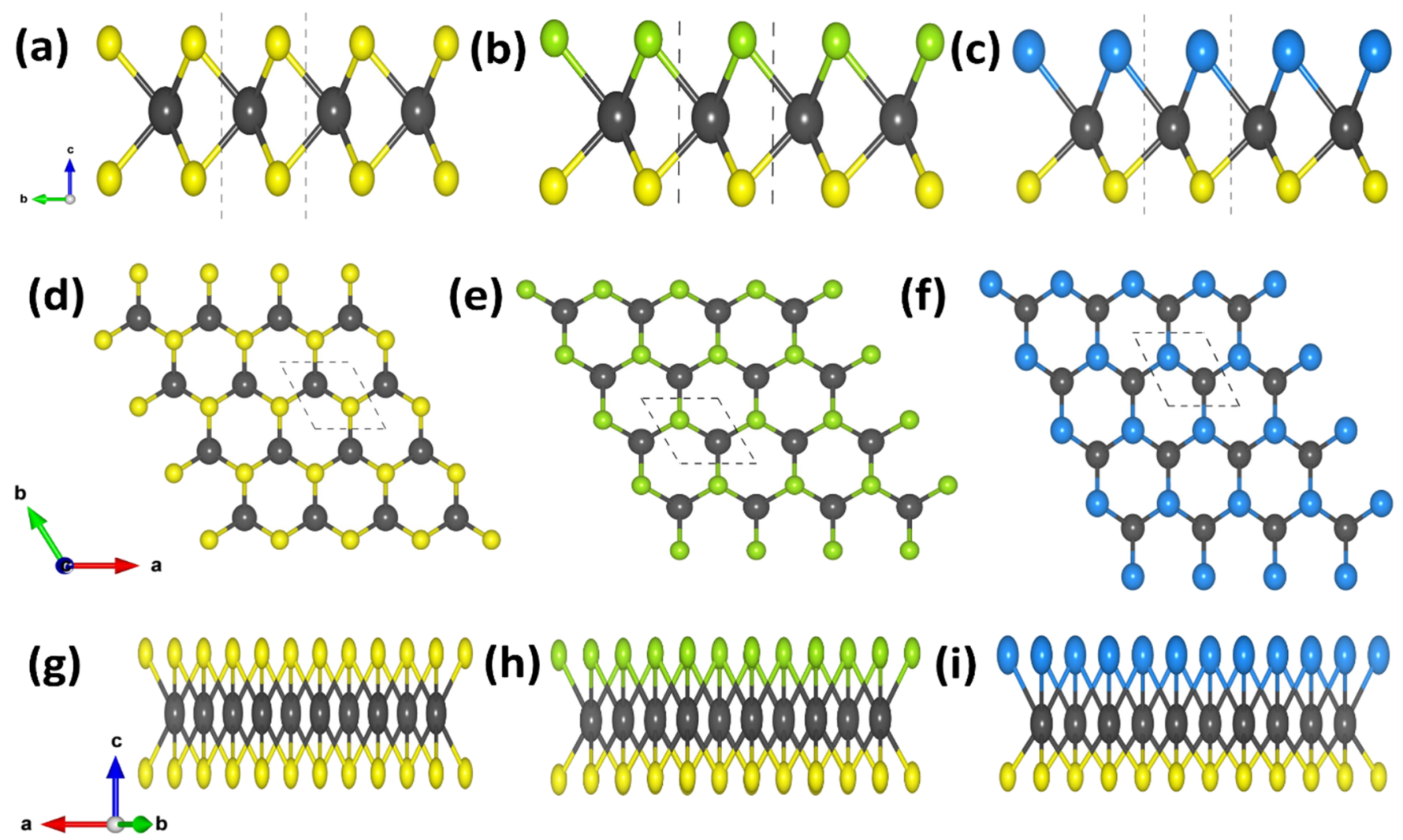

Figure 1. Unit cell of full optimized structures of (a) WS $\mathrm{W}_{2}$, (b) WSSe, and (c) WSTe. Top view of (d) WS ${ }_{2}$, (e) WSSe, and (f) WSTe, and also different orientations of the side view of $(\mathrm{g}) \mathrm{WS}_{2}$, (h) WSSe, and (i) WSTe Janus materials.

theoretical investigation by Cheng et al. ${ }^{18}$ Various methods, such as micromechanical exfoliation, liquid exfoliation, chemical vapor deposition (CVD), and hydrothermal synthesis, are used for the experimental synthesis of $2 \mathrm{D}$ materials. Janus $2 \mathrm{D}$ materials do not exist in nature. However, the Janus MoSSe monolayer from $\mathrm{MoS}_{2}$ was first successfully synthesized through CVD by $\mathrm{Li}$ and co-workers in 2017 . $^{16}$ The Janus monolayer MoSSe was also experimentally synthesized from $\mathrm{MoSe}_{2}$ in 2017. ${ }^{19}$ Among these previously reported materials, MoSSe provides a foundation for theoretical and experimental investigations on other Janus type materials. ${ }^{17}$ Recent theoretical studies suggest that the Janus monolayer has promising applications in various fields such as gas sensing, ${ }^{20}$ optoelectronics, ${ }^{21}$ photocatalysts, ${ }^{22,23}$ spintronics, and valleytronics. $^{18,24,25}$ In addition, Gu and Yang found that the lattice thermal conductivity can be significantly reduced by the minimization of the frequency gap. ${ }^{26}$ It can also be reduced by changing its stoichiometry. ${ }^{27,28}$ Low lattice thermal conductivity can effectively increase the ZT.

Motivated by these exciting properties of the Janus monolayer, we have systematically investigated the structural properties, electronic properties, lattice thermal conductivity, and thermoelectric behavior of the Janus 2D material WS-X (where $X=\mathrm{Se}$ and Te). The structure of WSSe and WSTe can be prepared by the replacement of one $S$ atom with Se and Te in the molecule of $\mathrm{WS}_{2}{ }^{27,29,30}$. We have also reported a comparative study with its traditional disulfide form $\mathrm{WS}_{2}$. To effectively simulate their thermoelectric behavior, the Seebeck coefficient, electric conductivity, thermal conductivity, and ZT have been determined as a function of temperature. The results of this study show the enhancement in thermoelectric performance from $\mathrm{WS}_{2}$ toward WSSe and WSTe.

\section{COMPUTATIONAL METHODS}

In this work, first-principles simulations are performed using Vienna $\mathrm{Ab}$ initio Simulation Package (VASP) software. ${ }^{31,32}$ The densityfunctional theory calculations are performed by the generalized gradient approximation method with the Perdew-Burke-Ernzerhof (PBE) exchange-correlation (XC) functional. ${ }^{33}$ For the monolayer WS-X system ( $X=S, S e$, and Te), a projected augmented wave (PAW) with a default energy of $500 \mathrm{eV}$ was used to describe the core and valence electron interactions. ${ }^{34}$ We also have the HeydScuseria-Ernzerhof (HSE06) functional to obtain accurate electronic properties. For hybrid functional HSE06, we have used the exchangecorrelation energy functional HSE06 with a mixing parameter $(\alpha)$ of $25 \%$ and a screening parameter of $0.2 \AA^{-1}$. The thick vacuum of order $25 \AA$ is inserted to prevent interactions between periodic layers along the normal direction (z-direction). ${ }^{35}$ The Monkhorst pack meshes of $(19 \times 19 \times 1)$ are used for the k-point sampling. ${ }^{36}$ For these materials, the optimized structures are achieved by the conjugate gradient (CG) method within a converge criterion of $10^{-3} \mathrm{eV} / \AA$ for Hellmann-Feynman forces and $10^{-8} \mathrm{eV} /$ cell for energy. In order to check dynamic stability, the phonon dispersions for these materials are calculated through the density functional perturbation theory (DPFT) method by creating supercells of $5 \times 5 \times 1$ and using a kpoint mesh of $3 \times 3 \times 1 .^{37}$ Lattice thermal conductivity of these materials are determined by a relaxation time approximation (RTA) method through a Phono3py code. ${ }^{38}$ The second-order force constants (for harmonic phonons) for the $4 \times 4 \times 1$ sized supercells of these materials and the third-order force (an-harmonic force constants) for the $3 \times 3 \times 1$ sized supercells of these materials are computed by the finite-displacement method using a k-point mesh of for the $3 \times 3 \times 1$ sized supercells of these materials. ${ }^{37}$ To calculate the lattice thermal conductivity, a q-mesh of $19 \times 19 \times 1$ is used for sampling. The alternative $24 \times 24 \times 1 \mathrm{q}$ mesh did not have a relevant effect on our conclusions. The deformation potential (DP) theory developed by Bardeen and Shockley is employed to calculate carriers' mobility. ${ }^{39}$ The semiclassical Boltzmann theory, Boltzmann transport 
Table 1. Calculated Lattice Parameters and Angles in Janus Materials

\begin{tabular}{lcccccc}
\multicolumn{1}{c}{ materials } & lattice & $a(\AA)$ & $b(\AA)$ & $\alpha$ & $\beta$ & $\gamma$ \\
WS $_{2}$ (monolayer) & hexagonal & 3.191 & 3.191 & $90^{\circ}$ & $90^{\circ}$ & $120^{\circ}$ \\
WSSe (JTMDs) & hexagonal & 3.258 & 3.258 & $90^{\circ}$ & $90^{\circ}$ & $120^{\circ}$ \\
WSTe (JTMDs) & hexagonal & 3.370 & 3.370 & $90^{\circ}$ & $90^{\circ}$ & $120^{\circ}$ \\
\hline
\end{tabular}
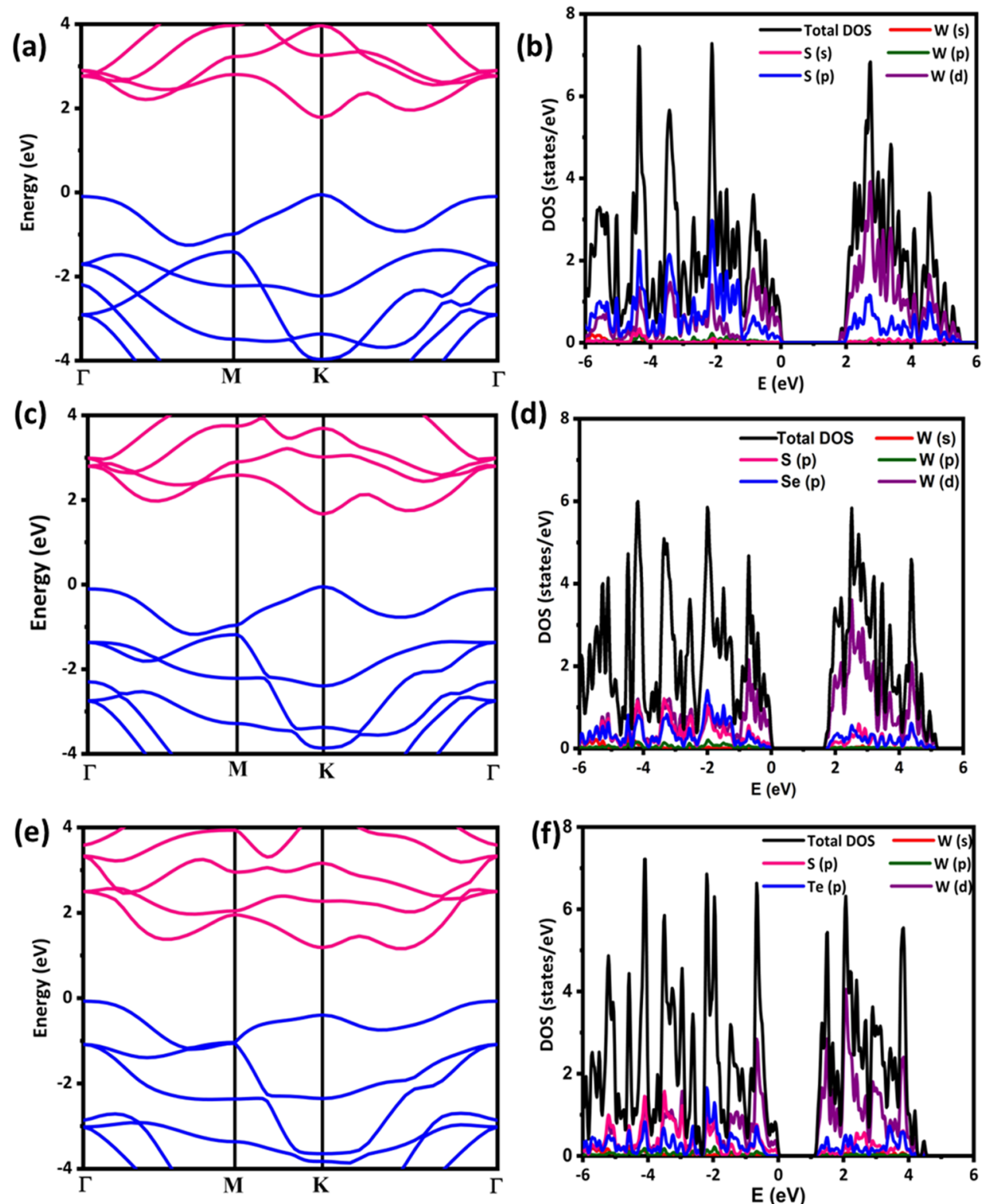

Figure 2. (a) Band structure of monolayer $\mathrm{WS}_{2}$, (b) PDOS of monolayer $\mathrm{WS}_{2}$, (c) band structure of Janus monolayer WSSe, (d) PDOS of Janus monolayer WSSe, (e) band structure of Janus monolayer WSTe, and (f) PDOS of monolayer WSTe.

equation (BTE), and RTA methods have been employed to calculate the Seebeck coefficient and transport properties of these materials through the BoltzTrap2 code. ${ }^{40,41}$ We have used constant relaxation time approximation with $t=10^{-14} \mathrm{~s}$ for thermal and electrical conductivity calculations. $\mathrm{Ab}$ initio molecular dynamics (AIMD) simulations ${ }^{31,42}$ were performed in the canonical ensemble (fixed particle number, volume, and temperature, NVT) with a time step of $2 \mathrm{fs}$ by keeping the temperature fixed at $1200 \mathrm{~K}$ to check the thermal stability. Vesta software was used for visualization of materials. ${ }^{43}$

\section{RESULTS AND DISCUSSIONS}

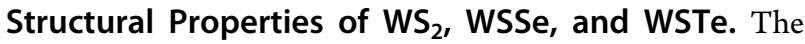
optimized stable structures of the monolayer $\mathrm{WS}_{2}$ and the
Janus monolayer WSSe and WSTe are shown in Figure 1. These materials have hexagonal lattice symmetry in $2 \mathrm{D}$ space. Here, each unit cell of these material contains three atoms (one atom of $\mathrm{W}$, one atom of $\mathrm{S}$, and one atom of $X=\mathrm{S}$, Se, or $\mathrm{Te})$. Each tungsten $(\mathrm{W})$ atom has four coordinates $(4 \mathrm{~W}-\mathrm{X}$ bonds; $X=S$, Se, and Te). While sulfur (S), selenium (Se), and tellurium $(\mathrm{Te})$ atoms have two coordinates $(2 \mathrm{~W}-\mathrm{X}$ bonds; $X=\mathrm{S}$, Se, or Te) in the structures. The optimized $\mathrm{WS}_{2}$ monolayer has lattice parameters $a=3.191 \AA$ and $b=3.191 \AA$ in $\mathrm{x}$ and $\mathrm{y}$ directions, respectively, as shown in Table 1 . This value is agreed with previously reported values. ${ }^{44,45} \mathrm{~A}$ high 
vacuum of $25 \AA$ is also present in the z-direction to prevent interactions between adjacent layers.

The Janus monolayer structure for this two-dimensional $\mathrm{WS}_{2}$ monolayer can be achieved by the replacement of any one $\mathrm{S}$ atom with Se atom or $\mathrm{Te}$ atom in its structure. After optimization of the predicated Janus materials WSSe and WSTe, the WSSe has lattice constants $a=3.258 \AA$, and $b=$ $3.258 \AA$ in $\mathrm{x}$ and $\mathrm{y}$ directions, respectively, and the WSTe has lattice constants $a=3.370 \AA$ and $b=3.370 \AA$ (see Table 1$)$. This optimized lattice parameter is very close to previous investigations. $^{27}$ Both materials, WSSe and WSTe, have a hexagonal lattice arrangement in $2 \mathrm{D}$ space. There are two $\mathrm{W}-$ $S$ bonds having a bond length of $2.42 \AA$, whereas the Janus monolayer WSSe has one $\mathrm{W}-\mathrm{S}$ bond of $2.55 \AA$ and another $\mathrm{W}-\mathrm{Se}$ bond of $2.43 \AA$. In addition, Janus WSTe has one W-S bond of $2.44 \AA$ and a $\mathrm{W}-\mathrm{Te}$ bond of $2.73 \AA$ as presented in Table 1 . The $\mathrm{W}-\mathrm{S}$ bonding is relatively weaker than that in the Janus TMDs, WSSe and WSTe. The W-Te bond in Janus WSTe is weaker than any other bonding among these materials. To check the dynamical stability of this monolayer, we have computed the phonon band structure as shown in Figure S1 (see Supporting Information). From the phonon band structures, we can see that there is no negative frequency. It means that the Janus monolayer is a dynamically stable structure. From ab initio molecular dynamics (AIMD) calculations, all these monolayers at $1200 \mathrm{~K}$ show thermal stability, which is shown in Figure S2 (see Supporting Information).

Electronic Properties of Monolayer $\mathrm{WS}_{2}$ and Janus WSSe and WSTe. To study the electronic properties of the $2 \mathrm{D}$ single materials $\mathrm{WS}_{2}$, WSSe, and WSTe, the PBE band structure and the hybrid band structure were computed along the symmetry path $\Gamma-\mathrm{M}-\mathrm{K}-\Gamma$. Here, Figure $2(\mathrm{a}, \mathrm{c}$, and e) shows the PBE bandstructure of monolayers $\mathrm{WS}_{2}$, WSSe, and WSTe. The calculated band gap of $\mathrm{WS}_{2}$ is $1.84 \mathrm{eV}$, which is of direct nature. This value of the direct band gap for the monolayer $\mathrm{WS}_{2}$ is very close to the experimental value of the band gap in the previous investigation. ${ }^{46-50}$ Similarly, the calculated directgaps of WSSe and WSTe are 1.72 and $1.23 \mathrm{eV}$, which are also agreed with earlier reported values. ${ }^{51}$ The band gaps for the HSE06 functional (see Figure S3 in Supporting Information) are $2.15 \mathrm{eV}$ for WSSe, which is very close to the previously reported values 2.07 and $1.71 \mathrm{eV}$ for WSTe, which also agrees with the pristine value $1.56 \mathrm{eV}^{30,52}$ The Janus TMD monolayers WSSe and WSTe have a semiconductor nature as presented in Table 2 .

For a better analysis of the electronic structure, the projected density of states (PDOS) for two-dimensional materials $\mathrm{WS}_{2}$, WSSe, and WSTe is determined as shown in Figure 2(b,d, and $\mathrm{f})$. For these materials, the $\mathrm{d}$-orbital of the $\mathrm{W}$ atom provides the main contribution to the formation of conduction bands. It

Table 2. Direct Band Gap (eV) at the K-Point of Materials Using PBE and HSE06 Functionals

$\begin{array}{clccc}\text { materials } & \text { material type } & \text { structure } & \begin{array}{c}E_{\mathrm{g}} \text { (using } \\ \mathrm{PBE})\end{array} & \begin{array}{c}E_{\mathrm{g}} \text { (using } \\ \text { HSE06) }\end{array} \\ \mathrm{WS}_{2} & \text { monolayer } & \begin{array}{c}\text { hexagonal } \\ (2 \mathrm{D})\end{array} & 1.83 & 2.78 \\ \text { WSSe } & \begin{array}{c}\text { Janus } \\ \text { monolayer }\end{array} & \begin{array}{c}\text { hexagonal } \\ (2 \mathrm{D})\end{array} & 1.72 & 2.15 \\ \text { WSTe } & \begin{array}{c}\text { Janus } \\ \text { monolayer }\end{array} & \begin{array}{c}\text { hexagonal } \\ (2 \mathrm{D})\end{array} & 1.23 & 1.71\end{array}$

also provides the main contribution in the valence band near the Fermi levels of these materials. The p-orbital of the $S$ atom also provides small contributions over conduction bands. Similarly, p-orbitals of S, Se, and Te have contributed to the conduction band of WSSe and WSTe. The s-orbitals of W, S, $\mathrm{Se}$, and $\mathrm{Te}$ have negligible contribution information of conduction and valence bands. Far from the Fermi level, the p-orbitals of $S, S e$, and Te have a more massive contribution in the valence band of Janus WSSe and WSTe. In the $\mathrm{WS}_{2}$ monolayer, this contribution has been provided by only the $S$ atom. This indicates the stable replacement of the $S$ atom by the Se and Te atom in Janus WSSe and WSTe, respectively.

Another significant contribution of d-orbitals of the $\mathrm{W}$ atom is found in the valence band of all of these materials. At many places, the d-orbitals of the $\mathrm{W}$ atom are overlapped with $\mathrm{p}$ orbitals of S, Se, and Te atoms in the valence band far from the Fermi level. The flat band lines in these suggest a large effective mass for them, and the Seebeck coefficient directly depends on the effective mass $\left(m^{*}\right) .{ }^{53}$ According to that, the monolayers $\mathrm{WS}_{2}$, Janus WSSe, and WSTe have higher values of the Seebeck coefficient.

Carrier Mobility and Relaxation Time. Using the DP theory based on the effective mass of carriers presented by Bardeen and Shockley, the carrier mobility can be determined by the following expression: $\mu=\frac{2 \mathrm{e} \hbar^{3} \mathrm{C}}{3 k_{B} T\left|m^{*}\right|^{2} E_{1}^{2}}$, where $\mu$ is carrier mobility, $C$ is elastic modulus in the strain direction, $m^{*}$ is the effective mass of the carrier, $k_{\mathrm{b}}$ is the Boltzmann constant, and $E_{1}$ is the DP. Here, the effective mass $m^{*}=\hbar^{2}\left[\frac{\partial^{2} E(k)}{\partial k^{2}}\right]^{-1}$ is obtained by parabolic fitting of the band edge, where $E(k)$ is the total energy and DP is defined as $E_{1}=$ $\frac{\partial E_{\text {edge }}}{\partial \delta}=\frac{\Delta \mathrm{CBM}(\mathrm{eV}) \text { or } \Delta \mathrm{VBM}(\mathrm{eV})}{\Delta \text { strain }(\%)}$, where $\Delta \mathrm{CBM}(\mathrm{eV})=\partial E_{\text {edge }}$ is the shift in the conduction band minimum and $\triangle \mathrm{VBM}(\mathrm{eV})=$ $\partial E_{\text {edge }}$ is the shift in the valence band minimum under the strain $\delta . E_{1}$ is determined by the linear fitting of the $\triangle \mathrm{CBM}$ $(\mathrm{eV})$ and $\triangle \mathrm{VBM}(\mathrm{eV})$ values of $E_{\text {edge }}$ with respect to strain $\mathrm{d}$ ( $\triangle \mathrm{CBM}$ for electrons and $\triangle \mathrm{VBM}$ for holes).

The strain $\delta(\%)$ is expressed as $\delta=\left[\frac{\left|a-a_{0}\right|}{a_{0}}\right] \times 100$. Here, $a_{0}$ is the lattice constant without strain and $a$ is the lattice constant after application of strain (see Figures S4-S6 in the Supporting Information).

Figure $3(\mathrm{a}, \mathrm{b})$ shows the plot of electron mobility and hole mobility. The electronic mobility of $\mathrm{WS}_{2}$ is slightly bigger than WSSe and WSTe. The effective masses of carriers in WSTe are slightly more than $\mathrm{WS}_{2}$ and WSTe as presented in Table 3. For $\mathrm{WS}_{2}$ and WSSe, the hole's effective mass is slightly larger than that of the electron in the case of WSTe as shown in Table 3, whereas the hole mobility of WSSe is slightly larger than $\mathrm{WS}_{2}$ and WSTe. The curve of carries' mobility has shown a parabolic decrease with temperature. In addition, the values of carrier mobilities at room temperature for Janus materials are presented in Table 3. In these materials, the relaxation time of the electron and can be calculated through the formula $\tau$ $=\frac{\mu m^{*}}{e}$. Therefore, the curve of relaxation time is also a parabolic function of temperature. The relaxation time of electronic collision is decreased with an increase in the temperature. The relaxation time of the carrier is the same for the $\mathrm{WS}_{2}$ monolayer and its Janus materials. Figure 3(c,d) shows the relaxation time of electrons and holes. 

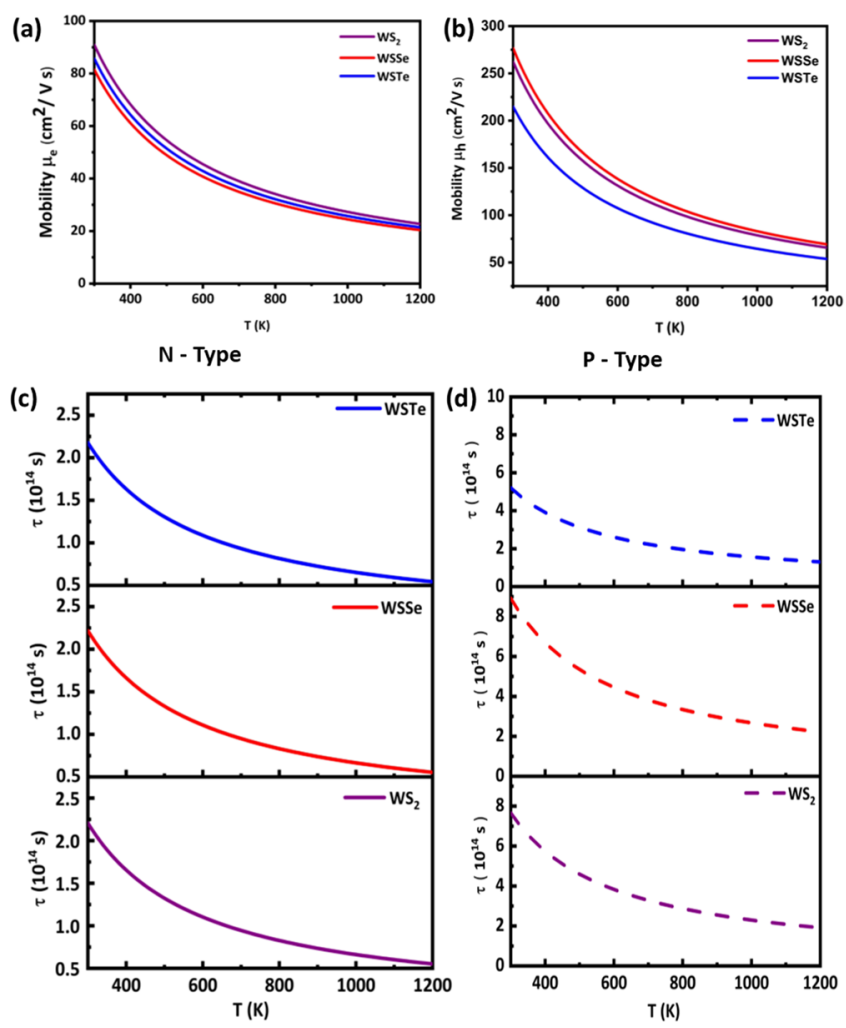

Figure 3. (a) Electron mobility, (b) hole mobility, (c) relaxation time of electrons, and (d) relaxation time of holes.

Table 3. Calculated Deformation Potential $\left(E_{1}\right)$, Elastic Constant $(\mathrm{C})$, the Effective Mass of the Electron $\left(m_{\mathrm{e}}{ }^{*}\right)$, the Effective Mass of the Hole $\left(m_{\mathrm{h}}{ }^{*}\right)$, Electron Mobility $\left(\mu_{\mathrm{e}}\right)$, and Hole Mobility $\left(\mu_{\mathrm{h}}\right)$ at $300 \mathrm{~K}$

\begin{tabular}{|c|c|c|c|c|c|}
\hline materials & carriers & $\begin{array}{c}E_{1} \\
(\mathrm{eV})\end{array}$ & $\begin{array}{c}C(\mathrm{~N} / \\
\mathrm{m})\end{array}$ & $m^{*}\left(\mathrm{~m}_{0}\right)$ & $\left.\mathrm{cm}^{2} \mathrm{~V}^{-1} \mathrm{~s}^{-1}\right)$ \\
\hline $\begin{array}{l}\mathrm{WS}_{2} \\
\quad \text { (monolayer) }\end{array}$ & $\begin{array}{l}\text { electron } \\
\text { hole }\end{array}$ & $\begin{array}{r}14.22 \\
6.96\end{array}$ & 235.46 & $\begin{array}{l}\mathrm{m}_{\mathrm{e}}^{*}=0.43 \\
\mathrm{~m}_{\mathrm{h}}^{*}= \\
\quad-0.52\end{array}$ & $\begin{array}{l}\mu_{\mathrm{e}}=90.97 \\
\mu_{\mathrm{h}}=262.36\end{array}$ \\
\hline WSSe (Janus) & $\begin{array}{l}\text { electron } \\
\text { hole }\end{array}$ & $\begin{array}{r}13.21 \\
6.06\end{array}$ & 229.31 & $\begin{array}{c}\mathrm{m}_{\mathrm{e}}^{*}=0.48 \\
\mathrm{~m}_{\mathrm{h}}^{*}=- \\
0.57\end{array}$ & $\begin{array}{l}\mu_{\mathrm{e}}=81.50 \\
\mu_{\mathrm{h}}=276.64\end{array}$ \\
\hline WSTe (Janus) & $\begin{array}{l}\text { electron } \\
\text { hole }\end{array}$ & $\begin{array}{r}13.59 \\
8.99\end{array}$ & 222.21 & $\begin{array}{l}\mathrm{m}_{\mathrm{e}}^{*}=0.45 \\
\mathrm{~m}_{\mathrm{h}}^{*}= \\
-0.43\end{array}$ & $\begin{array}{l}\mu_{\mathrm{e}}=85.73 \\
\mu_{\mathrm{h}}=214.79\end{array}$ \\
\hline
\end{tabular}

Thermoelectric Properties of Janus Monolayer Materials. The Seebeck coefficient, electric conductivity, thermal conductivity, and ZT are calculated as a function of temperature with a constant relaxation time $\left(\tau=10^{-14} \mathrm{~s}\right)$ for the monolayer $\mathrm{WS}_{2}$ and the Janus monolayers WSSe and WSTe. Figure 4(a) shows the computed Seebeck coefficients of the material at a temperature range from 300to $1200 \mathrm{~K}$. Initially, the Seebeck coefficient of a pure monolayer $\mathrm{WS}_{2}$ has a slightly higher value than both Janus monolayers at $300 \mathrm{~K}$ (see Table 4). For the materials $\mathrm{WS}_{2}$, WSSe, and WSTe, the Seebeck effect decreases as a parabolic function of temperature. In the Janus monolayers WSSe and WSTe, the value of Seebeck coefficients decreases more slowly than the pure monolayer $\mathrm{WS}_{2}$. The value of Janus WSSe Seebeck coefficients exceeds the values of $\mathrm{WS}_{2}$ near $400 \mathrm{~K}$. Similarly, the curve of the Seebeck coefficient of WSTe crosses at a higher temperature at $800 \mathrm{~K}$. At higher temperatures, both Janus materials WSSe and WSTe have shown more Seebeck effect.

Figure 4(b) shows the electrical conductivity of materials. The Janus monolayer WSTe has low electric conductivity than the monolayer $\mathrm{WS}_{2}$ and the Janus monolayer WSSe in the whole temperature range. The electric conductivity of all materials increases with temperature, but the electric conductivity of WSTe increases much slower than $\mathrm{WS}_{2}$ and WSSe. For higher temperatures, $\mathrm{WS}_{2}$ and WSSe have almost the same value. At low temperatures, the electric conductivity of WSSe shows a slightly higher value.

Thermal conductivities of the monolayer $\mathrm{WS}_{2}$ and the JTMD monolayers WSSe and WSTe are shown in Figure 4(df). The monolayer $\mathrm{WS}_{2}$ has higher thermal conductivity than the JTMD monolayers WSSe and WSTe. It is observed that the WSTe has ultralow thermal conductivity because both the electronic part and lattice contribution are very low; whereas, for the $\mathrm{WS}_{2}$ and WSSe, the lattice thermal conductivity is very high in comparison to the electronic thermal conductivity. Therefore, they too have a high thermal conductivity in comparison to WSTe. WSTe has ultralow thermal conductivity. Its value is almost 150 times smaller than that of $\mathrm{WS}_{2}$ and 100 times smaller than that of WSSe near room temperature. The thermal conductivity of the Janus WSTe monolayer is relatively very low as compared to other $2 \mathrm{D}$ materials. ${ }^{54-56}$ The ultralow value of thermal conductivity plays a significant role in the enhancement of the high ZT. Graphene has a high thermoelectric power factor $\left(S^{2} \sigma\right)$, but it has a very low $\mathrm{ZT}$ value of $0.55 \times 10^{-3}$ at room temperature due to its ultrahigh thermal conductivity. ${ }^{57}$ Graphene oxide is a potential $2 \mathrm{D}$ thermoelectric material with a $\mathrm{ZT}$ value of $0.05-$ 0.75 due to its thermopower factor of $127-287 \mu \mathrm{V} \mathrm{K}^{-1}(4-22$ times of the graphene value) and very low lattice thermal conductivity of $15-22 \%$ of the graphene value. ${ }^{58}$ Therefore, lattice thermal conductivity plays an important role in the thermoelectric ZT.

The calculated ZT values for these materials are shown in Figure $4(\mathrm{c}) . \mathrm{WS}_{2}$ and WSSe have low ZT values in the whole temperature range because both these materials have high thermal conductivity. The monolayer $\mathrm{WS}_{2}$ and the Janus monolayer WSSe report a ZT value of 0.006 and 0.012 . Here, it is observed that the replacement of one $S$ atom by a Se atom optimizes the ZT value. Predicated WSTe shows high thermoelectric conversion performance with a high $\mathrm{ZT}$ value. Initially, its $\mathrm{ZT}$ value is 0.75 at $300 \mathrm{~K}$ (near room temperature) as shown in Table 4. Its $\mathrm{ZT}$ increases with temperature. At higher temperatures (near $600 \mathrm{~K}$ temperature), it becomes constant with temperature. It shows $\mathrm{ZT}=2.56$ at a higher temperature $(1200 \mathrm{~K})$ as shown in Table 4 . The single layers of $\mathrm{GaS}, \mathrm{GaSe}$, and GaTe have ZT values of $0.89,0.90$, and 0.85 at room temperature, respectively, and decrease with the increase in temperature. ${ }^{59}$ The single-layer $\mathrm{MoS}_{2}$ has reported $\mathrm{ZT}$ values of $0.04,0.07$, and 0.11 at $300 \mathrm{~K}, 400 \mathrm{~K}$, and $500 \mathrm{~K}$, respectively. ${ }^{60}$ The $\mathrm{ZT}$ of $0.52,0.59$, and 0.25 at room temperature are found in AlS, AlSe, and AlTe monolayers under moderate conditions. ${ }^{61}$ The anisotropic materials $\mathrm{PdS}_{2}$, $\mathrm{PdSe}_{2}$ and $\mathrm{PdTe}_{2}$ show larger thermoelectric conversion performance with ZT values of $0.85,1.18$, and 2.42, respectively, compared to the commercial thermoelectric material $\mathrm{Bi}_{2} \mathrm{~S}_{3}(\mathrm{ZT}=0.80) .{ }^{55}$ 
(a)
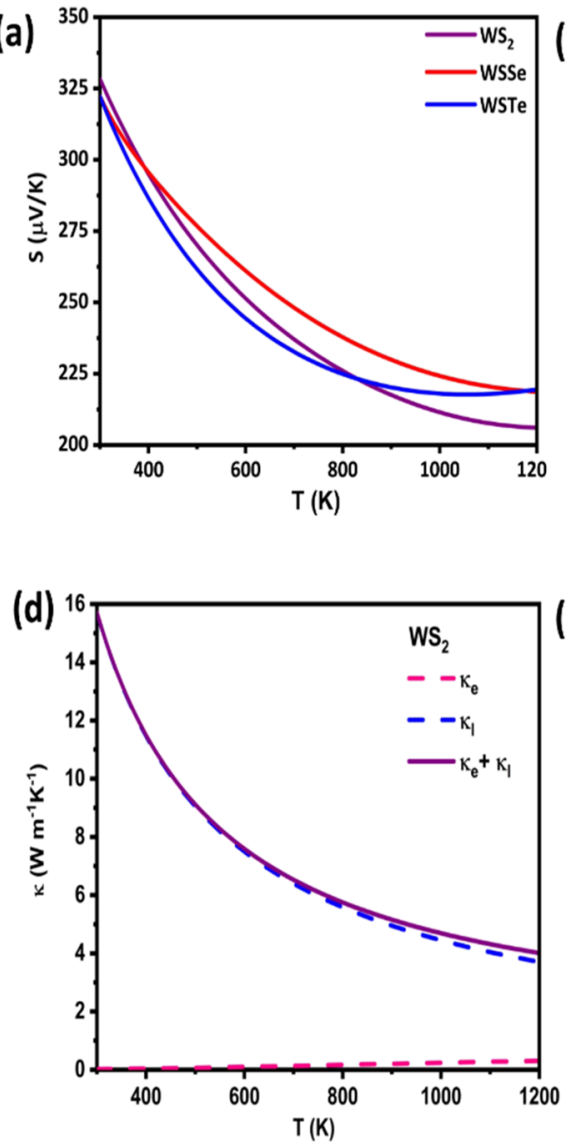
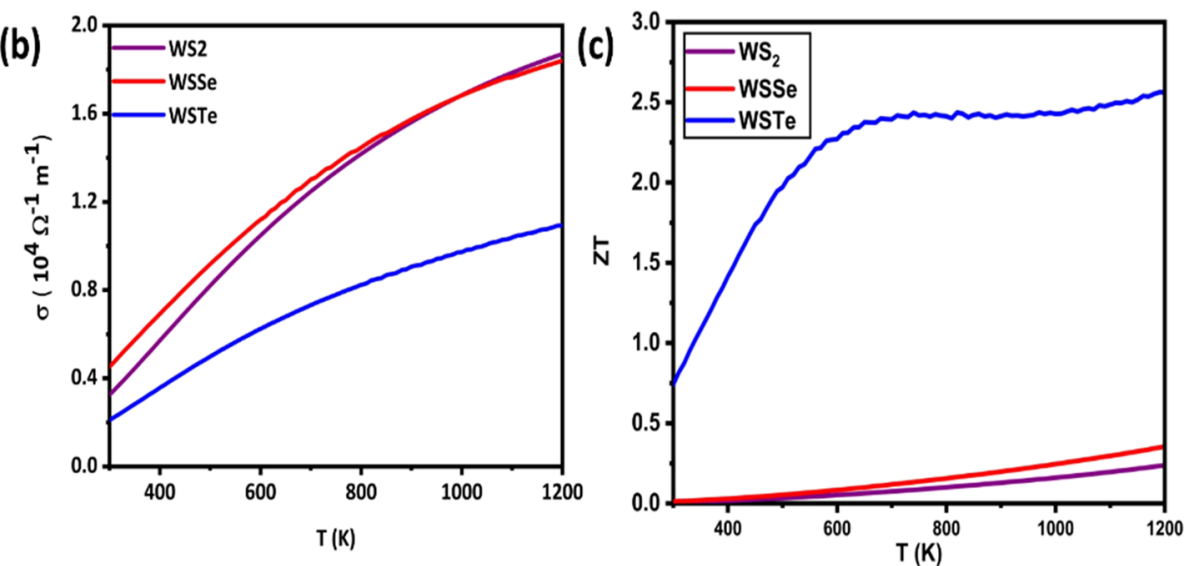

(e)

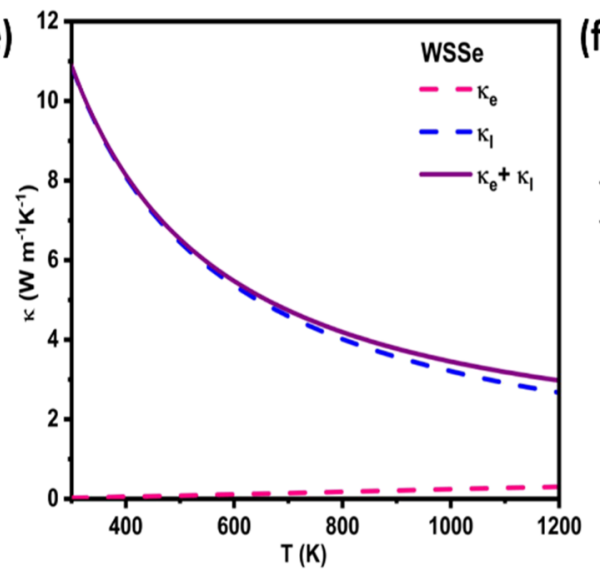

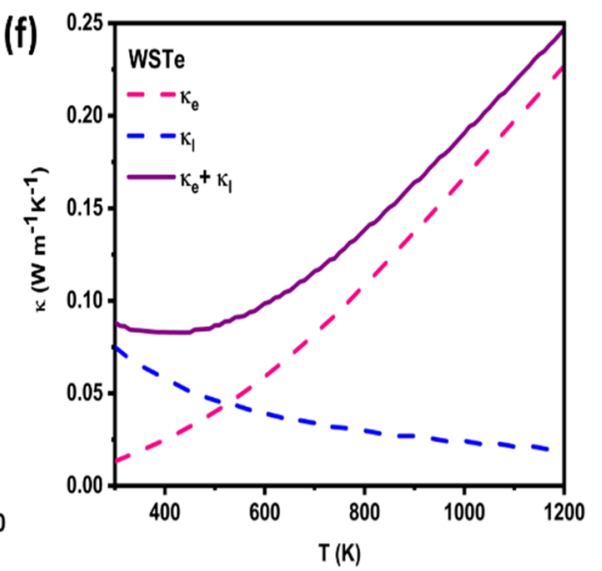

Figure 4. (a) Seebeck Coefficients, (b) electrical conductivity, (c) ZT values of monolayer $\mathrm{WS}_{2}$, Janus TMDs WSSe, and WSTe, (d) thermal conductivity of $\mathrm{WS}_{2}$, (e) thermal conductivity of WSSe, and (f) thermal conductivity of WSTe.

Table 4. Calculated Seebeck Coefficient (S) and Figure of Merit (ZT)

\begin{tabular}{lcccc} 
& \multicolumn{2}{c}{$\mathrm{S}(\mu \mathrm{V} / \mathrm{K})$} & \multicolumn{2}{c}{$\mathrm{ZT}$} \\
\multicolumn{1}{c}{ material } & $300 \mathrm{~K}$ & $1200 \mathrm{~K}$ & $300 \mathrm{~K}$ & $1200 \mathrm{~K}$ \\
WS $_{2}$ (monolayer) & 328.15 & 206.08 & 0.006 & 0.238 \\
WSSe (JTMDs) & 322.26 & 218.66 & 0.013 & 0.355 \\
WSTe (JTMDs) & 322.15 & 219.44 & 0.742 & 2.562 \\
\hline
\end{tabular}

\section{CONCLUSIONS}

In the present study, the structural, electronic, and thermoelectric properties of the $2 \mathrm{D}$ monolayer $\mathrm{WS}_{2}$ and Janus TMD materials WSSe and WSTe are calculated by first-principles calculations. From the phonon band structures and ab initio molecular dynamics calculations, the monolayer materials considered are dynamically and thermally stable. $\mathrm{WS}_{2}$, WSSe, and WSTe show a direct band gap of 1.32, 1.72, and $1.84 \mathrm{eV}$, respectively. The band through the HSE06 functional is found to be $2.15 \mathrm{eV}$ for WSSe and $1.71 \mathrm{eV}$ for WSTe. The carrier mobility of holes is relatively higher than the electrons. The values of the Seebeck coefficient $(S)$ of $\mathrm{WS}_{2}$, WSSe, and WSTe are $328.15,322.26$, and $322.15 \mu \mathrm{V} \mathrm{K} \mathrm{K}^{-1}$, respectively. Replacement of one $\mathrm{S}$ atom by $\mathrm{Se}$ and $\mathrm{Te}$ in the $\mathrm{WS}_{2}$ structure significantly reduces the lattice thermal conductivity. This reduction in thermal conductivity results in an improvement in the ZT value. The Janus WSTe monolayer shows superior ZT values of 0.74 and 2.56 at $300 \mathrm{~K}$ and $1200 \mathrm{~K}$, respectively, which is larger than most of the $2 \mathrm{D}$ materials, and it can be superior to the commercial $\mathrm{TE}$ material $\mathrm{Bi}_{2} \mathrm{Te}_{3}$.
Among these materials, the Janus monolayer WSTe can be proposed with a ZT value of 2.56 at $1200 \mathrm{~K}$ for thermoelectric applications such as the production of electricity from waste heat and Peltier cooling.

\section{ASSOCIATED CONTENT}

\section{Supporting Information}

The Supporting Information is available free of charge at https://pubs.acs.org/doi/10.1021/acsami.0c13960.

Electronic band structures using phonon dispersion spectra, ab initio molecular dynamics calculations (AIMD) at $1200 \mathrm{~K}$, and HSE06 functional and strainenergy and VBM/CBM edge positions (PDF)

\section{AUTHOR INFORMATION}

\section{Corresponding Authors}

Deobrat Singh - Condensed Matter Theory group, Materials Theory Division, Department of Physics and Astronomy, Uppsala University, Uppsala 751-20, Sweden; 이이.org/ 0000-0001-7246-8743; Email: deobrat.singh@physics.uu.se

P. B. Thakor - Department of physics, Veer Narmad South Gujarat University, Surat 395007, India; Email: pbthakor@ rediffmail.com

Rajeev Ahuja - Condensed Matter Theory group, Materials Theory Division, Department of Physics and Astronomy, Uppsala University, Uppsala 751-20, Sweden; Applied Materials Physics, Department of Materials Science and Engineering, Royal Institute of Technology (KTH), S-100 44 
Stockholm, Sweden; 이이이.org/0000-0003-1231-9994;

Email: rajeev.ahuja@physics.uu.se

\section{Authors}

Abhishek Patel - Department of physics, Veer Narmad South Gujarat University, Surat 395007, India; ㅇo orcid.org/00000001-5989-4625

Yogesh Sonvane - Advanced Materials Lab, Department of Applied Physics, S.V. National Institute of Technology, Surat 395007, India; 이이이.org/0000-0001-7319-2865

Complete contact information is available at:

https://pubs.acs.org/10.1021/acsami.0c13960

\section{Author Contributions}

The manuscript was written through the contributions of all authors. All authors have given approval to the final version of the manuscript.

\section{Notes}

The authors declare no competing financial interest.

\section{ACKNOWLEDGMENTS}

A.P. is thankful to the Council of Scientific and Industrial Research (CSIR), India, for his Junior Research Fellowship (File No: 09/1008(0003)/2019-EMR-1) and financial supports. D.S. and R.A. thank Olle Engkvists stiftelse (198-0390), Carl Tryggers Stiftelse for Vetenskaplig Forskning (CTS: 18:4), and Swedish Research Council (VR-2016-06014) for financial support. HPC2N and SNIC are acknowledged for the high-performance computing (HPC) facilities.

\section{REFERENCES}

(1) Yang, L.; Chen, Z. G.; Dargusch, M. S.; Zou, J. High Performance Thermoelectric Materials: Progress and Their Applications. Adv. Energy Mater. 2018, 8, 1701797.

(2) Perera, F. Pollution from Fossil-Fuel Combustion Is the Leading Environmental Threat to Global Pediatric Health and Equity: Solutions Exist. Int. J. Environ.Res. Public. Health 2018, 15, 16.

(3) Jiang, X.; Zhu, L.; Li, B.; Yao, K. Thermoelectric Properties of Monolayer $\alpha$-Te: Low Lattice Thermal Conductivity and Extremely High Dimensionless Figure of Merit. Phys. Lett. Sect. A Gen. At. Solid State Phys 2020, 384, 126222.

(4) Owusu, P. A.; Asumadu-Sarkodie, S. A Review of Renewable Energy Sources, Sustainability Issues and Climate Change Mitigation. Cogent Eng. 2016, 3, 1167990.

(5) Sorrell, S. Reducing Energy Demand: A Review of Issues, Challenges and Approaches. Renew. Sustain. Energy Rev. 2015, 47, 74-82.

(6) Zhang, X.; Zhao, L. D. Thermoelectric Materials: Energy Conversion between Heat and Electricity. J. Mater. 2015, 1, 92-105.

(7) Freer, R.; Powell, A. V. Realising the Potential of Thermoelectric Technology: A Roadmap. J. Mater. Chem. C 2020, 8, 441-463.

(8) Liu, W.; Jie, Q.; Kim, H. S.; Ren, Z. Current Progress and Future Challenges in Thermoelectric Power Generation: From Materials to Devices. Acta Mater. 2015, 87, 357.

(9) Snyder, G. J.; Snyder, A. H. Figure of Merit ZT of a Thermoelectric Device Defined from Materials Properties. Energy Environ. Sci. 2017, 10, 2280-2283.

(10) Mishra, P.; Singh, D.; Sonvane, Y.; Ahuja, R. Two-Dimensional Boron Monochalcogenide Monolayer for Thermoelectric Material. Sustain. Energy Fuels 2020, 4, 2363-2369.

(11) Patel, A.; Singh, D.; Sonvane, Y.; Thakor, P. B.; Ahuja, R. Bulk and Monolayer As2S3 as Promising Thermoelectric Material with High Conversion Performance. Comput. Mater. Sci. 2020, 183, 109913.

(12) Beretta, D.; Neophytou, N.; Hodges, J. M.; Kanatzidis, M. G.; Narducci, D.; Martin-Gonzalez, M.; Beekman, M.; Balke, B.; Cerretti,
G.; Tremel, W.; et al. Thermoelectrics: From History, a Window to the Future. Mater. Sci. Eng. R Reports 2019, 138, 100501.

(13) Nshimyimana, E.; Hao, S.; Su, X.; Zhang, C.; Liu, W.; Yan, Y.; Uher, C.; Wolverton, C.; Kanatzidis, M. G.; Tang, X. Discordant Nature of $\mathrm{Cd}$ in $\mathrm{GeTe}$ Enhances Phonon Scattering and Improves Band Convergence for High Thermoelectric Performance. J. Mater. Chem. A 2020, 8, 1193-1204.

(14) Zhao, L. D.; Lo, S. H.; Zhang, Y.; Sun, H.; Tan, G.; Uher, C.; Wolverton, C.; Dravid, V. P.; Kanatzidis, M. G. Ultralow Thermal Conductivity and High Thermoelectric Figure of Merit in SnSe Crystals. Nature 2014, 508, 373-377.

(15) Hinterleitner, B.; Knapp, I.; Poneder, M.; Shi, Y.; Müller, H.; Eguchi, G.; Eisenmenger-Sittner, C.; Stöger-Pollach, M.; Kakefuda, Y.; Kawamoto, N.; et al. Thermoelectric Performance of a Metastable Thin-Film Heusler Alloy. Nature 2019, 576, 85-90.

(16) Li, R.; Cheng, Y.; Huang, W. Recent Progress of Janus 2D Transition Metal Chalcogenides: From Theory to Experiments. Small 2018, 14, 1802091

(17) Lu, A.-Y.; Zhu, H.; Xiao, J.; Chuu, C.-P.; Han, Y.; Chiu, M.-H.; Cheng, C.-C.; Yang, C.-W.; Wei, K.-H.; Yang, Y.; et al. Janus Monolayers of Transition Metal Dichalcogenides. Nat. Nanotechnol. 2017, 12, 744-749.

(18) Cheng, Y. C.; Zhu, Z. Y.; Tahir, M.; Schwingenschlögl, U. SpinOrbit-Induced Spin Splittings in Polar Transition Metal Dichalcogenide Monolayers. EPL (Europhysics Lett) 2013, 102, 57001.

(19) Zhang, J.; Jia, S.; Kholmanov, I.; Dong, L.; Er, D.; Chen, W.; Guo, H.; Jin, Z.; Shenoy, V. B.; Shi, L.; et al. Janus Monolayer Transition-Metal Dichalcogenides. ACS Nano 2017, 11, 8192-8198.

(20) Yang, X.; Singh, D.; Xu, Z.; Ahuja, R. Sensing the Polar Molecules $\mathrm{MH}_{3}(\mathrm{M}=\mathrm{N}, \mathrm{P}$, or As) with a Janus NbTeSe Monolayer. New J. Chem. 2020, 44, 7932-7940.

(21) Yang, X.; Singh, D.; Xu, Z.; Wang, Z.; Ahuja, R. An Emerging Janus MoSeTe Material for Potential Applications in Optoelectronic Devices. J. Mater. Chem. C 2019, 7, 12312-12320.

(22) Yang, X.; Banerjee, A.; Ahuja, R. Structural Insight of the Frailty of 2D Janus NbSeTe as An Active Photocatalyst. Chem CatChem 2020, DOI: $10.1002 /$ cctc. 202000540 .

(23) Rawat, A.; Mohanta, M. K.; Jena, N.; Dimple; Ahammed, R.; De Sarkar, A. Nanoscale Interfaces of Janus Monolayers of Transition Metal Dichalcogenides for 2D Photovoltaic and Piezoelectric Applications. J. Phys. Chem. C 2020, 124, 10385-10397.

(24) Yang, J.; Wang, A.; Zhang, S.; Liu, J.; Zhong, Z.; Chen, L. Coexistence of Piezoelectricity and Magnetism in Two-Dimensional Vanadium Dichalcogenides. Phys. Chem. Chem. Phys. 2019, 21, 132136.

(25) Ji, Y.; Yang, M.; Lin, H.; Hou, T.; Wang, L.; Li, Y.; Lee, S.-T. Janus Structures of Transition Metal Dichalcogenides as the Heterojunction Photocatalysts for Water Splitting. J. Phys. Chem. C 2018, 122, 3123-3129.

(26) Gu, X.; Yang, R. Phonon Transport in Single-Layer Transition Metal Dichalcogenides: A First-Principles Study. Appl. Phys. Lett. 2014, 105, 131903.

(27) Guo, S.-D.; Dong, J. Biaxial Strain Tuned Electronic Structures and Power Factor in Janus Transition Metal Dichalchogenide Monolayers. Semicond. Sci. Technol. 2018, 33, 85003.

(28) Tao, W.-L.; Mu, Y.; Hu, C.-E.; Cheng, Y.; Ji, G.-F. Electronic Structure, Optical Properties, and Phonon Transport in Janus Monolayer PtSSe via First-Principles Study. Philos. Mag. 2019, 99, $1025-1040$

(29) Idrees, M.; Din, H. U.; Ali, R.; Rehman, G.; Hussain, T.; Nguyen, C. V.; Ahmad, I.; Amin, B. Optoelectronic and Solar Cell Applications of Janus Monolayers and Their van Der Waals Heterostructures. Phys. Chem. Chem. Phys. 2019, 21, 18612-18621.

(30) Wang, J.; Shu, H.; Zhao, T.; Liang, P.; Wang, N.; Cao, D.; Chen, X. Intriguing Electronic and Optical Properties of TwoDimensional Janus Transition Metal Dichalcogenides. Phys. Chem. Chem. Phys. 2018, 20, 18571-18578. 
(31) Kresse, G.; Furthmüller, J. Efficient Iterative Schemes for Ab Initio Total-Energy Calculations Using a Plane-Wave Basis Set. Phys. Rev. B - Condens. Matter Mater Phys. 1996, 54, 11169-11186.

(32) Kresse, G.; Furthmüller, J.; Hafner, J. Ab Initio Force Constant Approach to Phonon Dispersion Relations of Diamond and Graphite. Europhys. Lett. 1995, 32, 729-734.

(33) Perdew, J. P.; Burke, K.; Ernzerhof, M. Generalized Gradient Approximation Made Simple. Phys. Rev. Lett. 1996, 77, 3865-3868.

(34) Kresse, G.; Joubert, D. From Ultrasoft Pseudopotentials to the Projector Augmented-Wave Method. Phys. Rev. B 1999, 59, 17581775.

(35) Chaurasiya, R.; Dixit, A.; Pandey, R. Strain-Mediated Stability and Electronic Properties of WS2, Janus WSSe and WSe $e_{2}$ Monolayers. Superlattices Microstruct. 2018, 122, 268-279.

(36) Monkhorst, H. J.; Pack, J. D. Special Points for Brillouin-Zone Integrations. Phys. Rev. B 1976, 13, 5188-5192.

(37) Togo, A.; Tanaka, I. First Principles Phonon Calculations in Materials Science. Scr. Mater. 2015, 108, 1-5.

(38) Mizokami, K.; Togo, A.; Tanaka, I. Lattice Thermal Conductivities of Two $\mathrm{SiO}_{2}$ Polymorphs by First-Principles Calculations and the Phonon Boltzmann Transport Equation. Phys. Rev. B 2018, 97, 224306.

(39) Bardeen, J.; Shockley, W. Deformation Potentials and Mobilities in Non-Polar Crystals. Phys. Rev. 1950, 80, 72-80.

(40) Madsen, G. K. H.; Carrete, J.; Verstraete, M. J. BoltzTraP2, a Program for Interpolating Band Structures and Calculating SemiClassical Transport Coefficients. Comput. Phys. Commun. 2018, 231, 140-145.

(41) Madsen, G. K. H.; Singh, D. J. BoltzTraP. A Code for Calculating Band-Structure Dependent Quantities. Comput. Phys. Commun. 2006, 175, 67-71.

(42) Kresse, G.; Hafner, J. Ab Initio Molecular Dynamics for Liquid Metals. Phys. Rev. B 1993, 47, 558-561.

(43) Momma, K.; Izumi, F. VESTA 3 for Three-Dimensional Visualization of Crystal, Volumetric and Morphology Data. J. Appl. Crystallogr. 2011, 44, 1272-1276.

(44) Amin, B.; Kaloni, T. P.; Schwingenschlögl, U. Strain Engineering of WS2, WSe2, and WTe2. RSC Adv. 2014, 4, 3456134565 .

(45) Kang, J.; Tongay, S.; Zhou, J.; Li, J.; Wu, J. Band Offsets and Heterostructures of Two-Dimensional Semiconductors. Appl. Phys. Lett. 2013, 102, 12111.

(46) Gusakova, J.; Wang, X.; Shiau, L. L.; Krivosheeva, A.; Shaposhnikov, V.; Borisenko, V.; Gusakov, V.; Tay, B. K. Electronic Properties of Bulk and Monolayer TMDs: Theoretical Study Within DFT Framework (GVJ-2e Method). Phys. status solidi 2017, 214, 1700218.

(47) Gutiérrez, H. R.; Perea-López, N.; Elías, A. L.; Berkdemir, A.; Wang, B.; Lv, R.; López-Urías, F.; Crespi, V. H.; Terrones, H.; Terrones, M. Extraordinary Room-Temperature Photoluminescence in Triangular $\mathrm{WS}_{2}$ Monolayers. Nano Lett. 2013, 13, 3447-3454.

(48) Elías, A. L.; Perea-López, N.; Castro-Beltrán, A.; Berkdemir, A.; Lv, R.; Feng, S.; Long, A. D.; Hayashi, T.; Kim, Y. A.; Endo, M.; et al. Controlled Synthesis and Transfer of Large-Area $\mathrm{WS}_{2}$ Sheets: From Single Layer to Few Layers. ACS Nano 2013, 7, 5235-5242.

(49) Zhao, W.; Ghorannevis, Z.; Chu, L.; Toh, M.; Kloc, C.; Tan, P.H.; Eda, G. Evolution of Electronic Structure in Atomically Thin Sheets of $\mathrm{WS}_{2}$ and $\mathrm{WSe}_{2}$. ACS Nano 2013, 7, 791-797.

(50) Tongay, S.; Fan, W.; Kang, J.; Park, J.; Koldemir, U.; Suh, J.; Narang, D. S.; Liu, K.; Ji, J.; Li, J.; et al. Tuning Interlayer Coupling in Large-Area Heterostructures with CVD-Grown $\mathrm{MoS}_{2}$ and $\mathrm{WS}_{2}$ Monolayers. Nano Lett. 2014, 14, 3185-3190.

(51) Liu, H.; Huang, Z.; He, C.; Wu, Y.; Xue, L.; Tang, C.; Qi, X.; Zhong, J. Strain Engineering the Structures and Electronic Properties of Janus Monolayer Transition-Metal Dichalcogenides. J. Appl. Phys. 2018, 125, 82516.

(52) Idrees, M.; Din, H. U.; Rehman, S. U.; Shafiq, M.; Saeed, Y.; Bui, H. D.; Nguyen, C. V.; Amin, B. Electronic Properties and Enhanced Photocatalytic Performance of van Der Waals Hetero- structures of $\mathrm{ZnO}$ and Janus Transition Metal Dichalcogenides. Phys. Chem. Chem. Phys. 2020, 22, 10351-10359.

(53) Levin, E. M. Charge Carrier Effective Mass and Concentration Derived from Combination of Seebeck Coefficient and ${ }^{125} \mathrm{Te}$ NMR Measurements in Complex Tellurides. Phys. Rev. B 2016, 93, 245202.

(54) Tao, W.-L.; Lan, J.-Q.; Hu, C.-E.; Cheng, Y.; Zhu, J.; Geng, H.Y. Thermoelectric Properties of Janus MXY ( $M=P d, P t ; X, Y=S$, Se, $\mathrm{Te})$ Transition-Metal Dichalcogenide Monolayers from First Principles. J. Appl. Phys. 2020, 127, No. 035101.

(55) Lan, Y.-S.; Chen, X.-R.; Hu, C.-E.; Cheng, Y.; Chen, Q.-F. Penta-PdX $\mathrm{PX}_{2}(\mathrm{X}, \mathrm{S}, \mathrm{Se}, \mathrm{Te})$ Monolayers: Promising Anisotropic Thermoelectric Materials. J. Mater. Chem. A 2019, 7, 11134-11142.

(56) Zhou, Y.; Zhao, Y.-Q.; Zeng, Z.-Y.; Chen, X.-R.; Geng, H.-Y. Anisotropic Thermoelectric Properties of Weyl Semimetal NbX (X = P and As): A Potential Thermoelectric Material. Phys. Chem. Chem. Phys. 2019, 21, 15167-15176.

(57) Anno, Y.; Imakita, Y.; Takei, K.; Akita, S.; Arie, T. Enhancement of Graphene Thermoelectric Performance through Defect Engineering. 2D Mater 2017, 4, 25019.

(58) Zhou, S.; Guo, Y.; Zhao, J. Enhanced Thermoelectric Properties of Graphene Oxide Patterned by Nanoroads. Phys. Chem. Chem. Phys. 2016, 18, 10607-10615.

(59) Bahuguna, B. P.; Saini, L. K.; Sharma, R. O.; Tiwari, B. Hybrid Functional Calculations of Electronic and Thermoelectric Properties of GaS, GaSe, and GaTe Monolayers. Phys. Chem. Chem. Phys. 2018, $20,28575-28582$.

(60) Jin, Z.; Liao, Q.; Fang, H.; Liu, Z.; Liu, W.; Ding, Z.; Luo, T.; Yang, N. A Revisit to High Thermoelectric Performance of SingleLayer $\mathrm{MoS}_{2}$. Sci. Rep. 2015, 5, 18342.

(61) Chen, X.; Huang, Y.; Liu, J.; Yuan, H.; Chen, H. Thermoelectric Performance of Two-Dimensional AlX $(X=S, S e, T e)$ : A First-Principles-Based Transport Study. ACS Omega 2019, 4, 1777317781. 